\title{
Phase defect inspection on EUV masks using RESCAN
}

\author{
Rajendran Rajeev*, Iacopo Mochi, Dimitrios Kazazis, Sara Fernandez, Tseng Li-Teng, \\ Patrick Helfenstein \& Yasin Ekinci, \\ Laboratory for Micro and Nanotechnology, \\ Paul Scherrer Institut, Villigen AG, Switzerland - 5232. \\ *rajeev.rajendran@psi.ch.
}

\begin{abstract}
As extreme ultraviolet (EUV) lithography is entering the high-volume manufacturing (HVM) phase, the ability to identify printable defects on EUV reticles becomes increasingly important to achieve the required wafer yield. However, no commercially available tool exists today for actinic patterned mask inspection (APMI). RESCAN is an APMI tool based on scanning coherent diffraction imaging (SCDI) under development at the Paul Scherrer Institut. In the last years, using RESCAN, we have demonstrated actinic identification of absorber defects on mask down to $36 \mathrm{~nm}$ size, and throughpellicle defect inspection. In this paper, we address a very critical but hitherto not reported feature of an APMI tool, namely the identification and characterization of phase defects on a patterned mask. Phase defects could be due to imperfections on the blank substrate leading to modification of the multilayer topology or due to particles embedded within the multilayer itself. Independent of the origin, the wave exiting the multilayer surface will have domains of phase variations as it propagates in the three-dimensional reticle stack. Mapping the exit wave that leave the EUV reticle both in amplitude and phase would be of paramount importance towards accurately predicting the EUV aerial images. Exploiting the amplitude and phase maps provided by SCDI, we use RESCAN for phase contrast imaging and to characterize programmed phase defects in a hybrid absorber-phase sample in a lens-less scheme, demonstrating the capability of the method and the tool.
\end{abstract}

Keywords: actinic patterned mask inspection, phase defects, metrology, EUV reticle, lensless imaging, coherent photons

\section{INTRODUCTION}

In the last few years, consistent improvement in the EUV source performance and improved resist materials have allowed the targeted wafer throughput (wafers printed per hour). While this itself is a remarkable feat for the industry, the development of an actinic defect inspection tool, that is sensitive both to absorber and phase defects on an EUV photomask, is still a necessity.

RESCAN (REflective mode EUV mask SCANnning lensless imaging tool) is a lens-free reticle defect metrology platform operational at the Paul Scherrer Institut in Switzerland, being developed to provide an effective method for actinic patterned mask inspection (APMI). RESCAN scans a reticle with a coherent EUV beam (see Fig. 1) and records the far-field diffraction patterns and reconstructs the complex amplitude of the reticle using iterative phase retrieval algorithms [1]. Over the last few years with this lens-less technique, also referred to as scanning coherent diffractive imaging (SCDI), we have (1) demonstrated actinic imaging of the complex exit wave on an EUV reticle [1], (2) mapped the absorber defects on EUV reticles in die-to-die and die-to-database modes [2], (3) demonstrated through pellicle imaging[3], (4) developed an EUV-optimized high-performance imaging detector for high throughput and high sensitivity operation modes [1] and (5) proposed a standalone solution for the fab integration of the tool with a bright compact EUV source [4].

In this paper, we demonstrate the capability of RESCAN to identify phase defects on a patterned EUV reticle. By using a reticle sample with programmed phase defects under the multilayer with a patterned arbitrary absorbing layer on top, RESCAN simultaneously maps the amplitude and phase of the wave exiting the EUV reticle, without the need for throughfocus imaging. We also demonstrate the identification of programmed phase defects on mask blanks. We believe that with this extended feature of phase contrast, RESCAN offers a technically complete and technologically comprehensive platform that caters to metrology of all relevant defect inspection modalities required from an effective actinic patterned mask inspection tool. In Section 2, we briefly describe the RESCAN tool prototype, the experiment and the phase retrieval 
algorithm. In Section 3 we describe the absorber-phase reticle sample and the patterning process flow used to make these hybrid samples. The results of imaging on the hybrid samples are presented in Section 4.

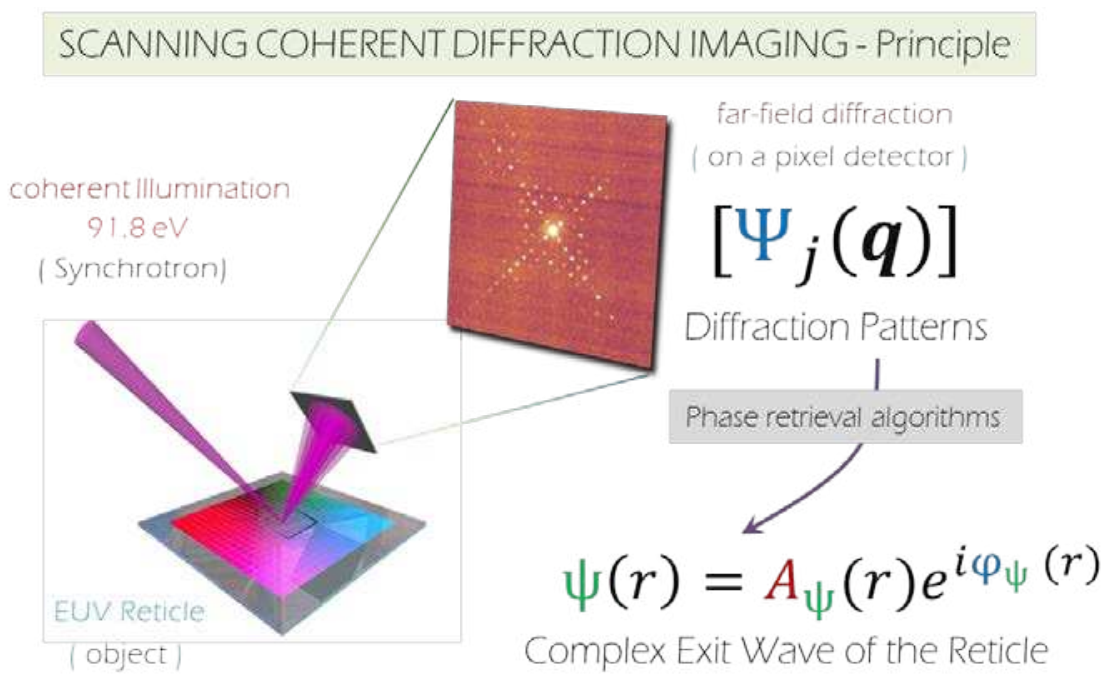

Figure 1. SCDI principle: The EUV reticle (object) is illuminated by coherent EUV photons and the far-field diffraction patterns are recorded on a pixel detector. Multiple diffraction patterns, $\Psi_{j}(q)$, collected at overlapping sites on the reticle allow computational phase retrieval and recovery of the exit wave $\psi(r)$ leaving the reticle both in amplitude $A_{\psi}(r)$ and phase $\varphi_{\psi}(r)$.

\section{METHODS}

RESCAN is based on SCDI in reflective mode [1]. Based on lens-less imaging, SCDI requires a simple measurement scheme using a pixel detector in the Fourier plane, and avoids complex optical components for both illumination and imaging. Experimentally, it consists of scanning the reticle with an EUV beam (referred to as probe), measuring the farfield diffraction intensity patterns and recovering the complex amplitude of the sample using phase retrieval algorithms [5-9,11] (see Fig. 3). The probe samples the reticle ensuring substantial overlap between adjacent illuminations, which creates information redundancy in the diffraction data. The data redundancy inherent in these measurements, allows the recovery of the complex object response function, despite the incomplete Fourier measurements due to the insensitivity of the detector to diffraction (spectral) phase.

Coherent diffractive imaging relies on the availability of bright sources of coherent photons. RESCAN uses an undulator at the Swiss Light Source (SLS) at Paul Scherer Institute. The undulator (Fig. 2a) emission in the EUV wavelength of concern $(13.5 \mathrm{~nm})$ is fully coherent spatially within a selected sub-aperture, but has a spectral bandwidth of $4 \%$ [10]. This leads to a smaller longitudinal coherence $L_{c o h}=\lambda^{2} / 2 \Delta \lambda$ of around $170 \mathrm{~nm}$ for $\lambda=13.5 \mathrm{~nm}$, insufficient to create quality diffraction patterns from the typical illumination spots of size $\sim 10 \mu \mathrm{m}$. To achieve the required longitudinal coherence for SCDI, the photons from the undulator are monochromatized using a spherical grating monochromator (SGM) (Fig. 2b) [10]. With the SGM, we achieve about $L_{c o h}=10 \mu \mathrm{m}$, allowing coherent illumination conditions over the typically used illumination spots $(\sim 10 \mu \mathrm{m})$. The monochromatic beam is then focused on the reticle sample surface as shown in Fig. 2c. The far-field diffraction patterns are collected by an EUV CCD. 


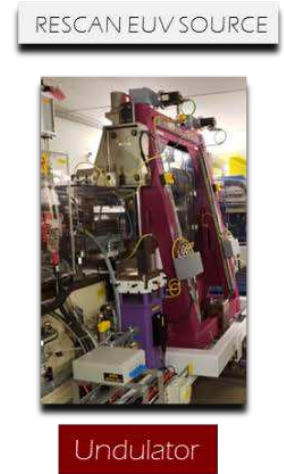

Bright coherent photons

$\lambda / \Delta \lambda \sim 20$

$\mathrm{P} \sim 100 \mathrm{~mW}$
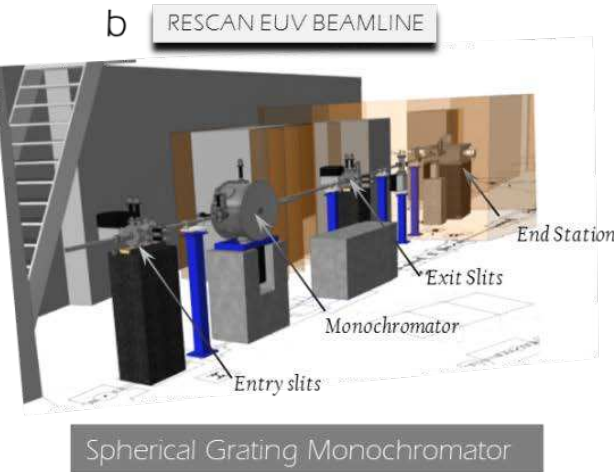

Enhanced longitudinal coherence

$\lambda / \Delta \lambda>1500$

$\mathrm{P} \sim 10 \mathrm{~mW}$

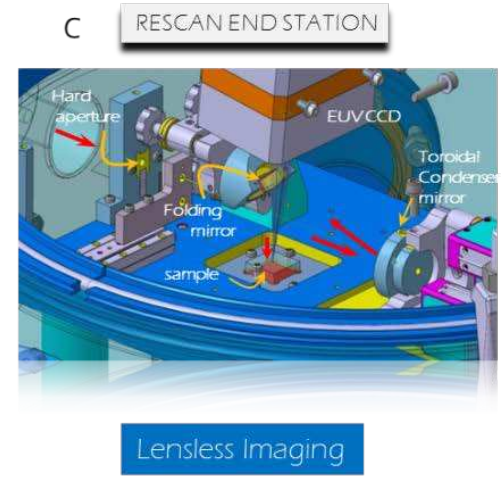

Simple Layout

NA 0.24

$\delta \sim 34 \mathrm{~nm}$

Figure 2. The RESCAN prototype tool: (a) The light source for RESCAN is an undulator source at the Swiss Light Source, tunable for soft x-ray photon energies between $60 \mathrm{eV}$ and $500 \mathrm{eV}$. At $91.8 \mathrm{eV}(13.5 \mathrm{~nm}$ ) the source bandwidth is $4 \%$. (b) SCDI requires high longitudinal coherence in additional to spatial coherence. To achieve this, a new beamline branch with a spherical grating monochromator was installed improving the monochromaticity by two orders of magnitude. (c) The end station for lens-less imaging of extended targets in reflective mode. The reticle sample is mounted on a stage below. The red lines indicate the optical path. The present configuration gives on-mask resolution of $34 \mathrm{~nm}$.

Presently, RESCAN is capable of handling reticle sample sizes of $20 \times 20 \mathrm{~mm}^{2}$ and the stage has a stroke of $200 \times 200 \mu \mathrm{m}^{2}$ with $5 \mathrm{~nm}$ accuracy in both axes. The illumination NA is varied by changing the entrance pupil diameter. For the experiments reported in this paper we used an illumination spot with a FWHM of $\sim 3 \mu \mathrm{m}$ and scan step size of $1 \mu \mathrm{m}$. The CCD that was used has a limited dynamic range that limited the resolution of the reconstructed image. To overcome this limitation, we stitched diffraction pattern images with different exposure times.

The set of exit waves, $\left\{\phi_{j}(r)\right\}$ (also referred to as state vector) is central to the phase retrieval algorithms used. The exit wave from the $j^{\text {th }}$ scan position is defined as the electromagnetic wave exiting the reticle surface which is essentially the probe $P(r)$ modulated by the response function $O(r)$ of the reticle (the object). With this, the exit waves at the $j^{\text {th }}$ scan position is defined as $\phi_{j}(r)=P\left(r-r_{j}\right) O(r)$, when the probe $P(r)$ is shifted to the position $r_{j}$ on the object $O(r)$.

Fourier inversion, $\mathfrak{F}^{-1}$, of the measured diffraction amplitudes $\Psi_{j}(q)=\mathfrak{F}\left[\phi_{j}(r)\right]$ to the sample plane cannot retrieve these complex functions $\phi_{j}(r)$, as the phase components $\Psi_{j}(q) /\left|\Psi_{j}(q)\right|$ are missing. However, the state vector $\left\{\phi_{j}(r)\right\}$ can still be recovered from the collective set of the measured diffraction intensities, $\left\{\Psi_{j}(q)\right\}$, provided there is substantial data redundancy in these measurements that can compensate for the lack of phase information in the Fourier plane. For this, SCDI uses phase retrieval algorithms based on the principle of Constraining and Intersection [11]. This is graphically described in Fig. 3a. By constraining the allowed solutions of the exit waves to two limited sub-sets of all possible solutions and by iterating between these subsets with a convergence-guaranteeing projection, the unique solution that lies in the intersection of these two subsets is solved [11]. The two constraining subsets for the exit waves are obtained from two independent but fundamental conditions intrinsic in measurements, namely that (1) the measured set of diffraction intensities match the computed far field equivalent of the exit waves and that (2) the exit waves from overlapping locations on the reticle are generated by shifting the probe across the same object. To arrive at the intersection of these two sets, in this study we used difference map iterative engine [9,11] ( see Fig. 3b). 
a
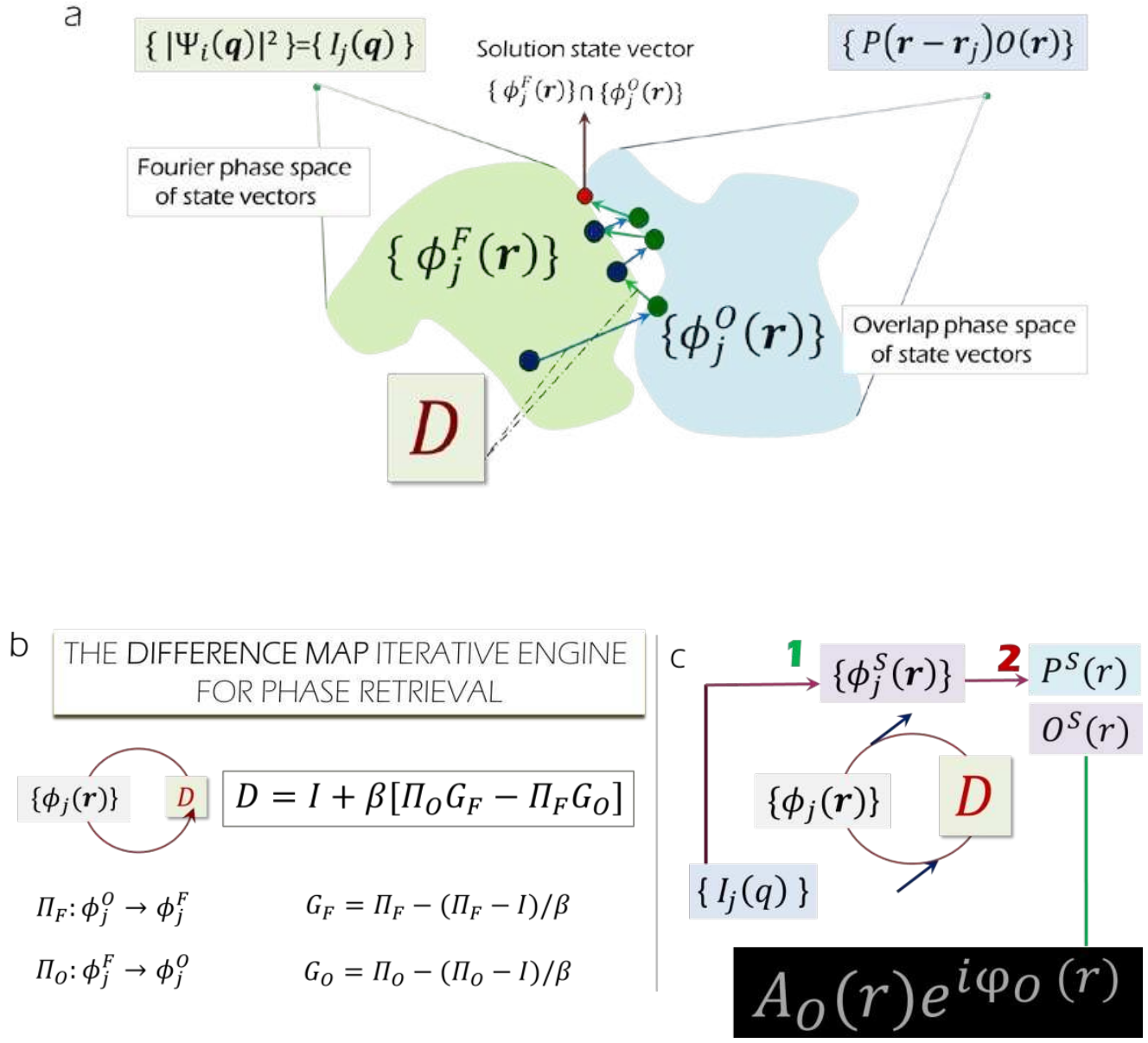

Figure 3. The difference map algorithm for phase retrieval in SCDI: (a) The solution for the set of exit waves indexed by the scan positions $j$ is determined by constraining the plausible solutions to two subsets $\left\{\phi_{j}^{F}(r)\right\}$ and $\left\{\phi_{j}^{O}(r)\right\}$ respectively referred to as Fourier projection space and overlap projection space of exit waves. The former essentially encompasses all possible solutions that are consistent with the Fourier intensity measurements and the latter forms a class functions $\phi_{j}^{O}(r)=$ $P\left(r-r_{j}\right) O(r)$, that is generated by shifting the probe $P(r)$ on the object $O(r)$ (which is essentially how the measurement are made). The unique solution set of exit waves, $\left\{\phi_{j}^{S}(r)\right\}$, lies in the intersection $\left\{\phi_{j}^{F}(r)\right\} \cap\left\{\phi_{j}^{O}(r)\right\}$ of these subsets and is obtained using an iterative projection map referred to as Difference Map $D[9,11]$. (b) The difference map D which by design avoids stagnation points in iterative solution search, is expressed in terms of the Fourier projection $\Pi_{F}: \phi_{j}^{O}(r) \Rightarrow \phi_{j}^{F}(r)$ and the overlap projection $\Pi_{O}: \phi_{j}^{F}(r) \Rightarrow \phi_{j}^{O}(r)$ maps for every iteration. For exclusive forms of $\Pi_{F}$ and $\Pi_{O}$ see [5,9]. (c) Summary of the difference map iterative engine for reticle imaging. The difference map engine takes in a redundant set of diffraction intensities $\left\{I_{j}(q)\right\}$, iteratively solve for the unique set of exit waves $\left\{\phi_{j}^{S}(r)\right\}$ and simultaneously decomposes the exit waves into the solution probe $P^{S}(r)$ and object $O^{S}(r)[8]$. The amplitude $A_{O}(r)$ and phase $\varphi_{O}(r)$ maps of the reticle response (object) is then retrieved from the complex function $O^{S}(r)=A_{O}(r) e^{i \varphi_{O}(r)}$.

One of the major advantages of the SCDI methods is that it allows recovery of the phase maps of the reticle along with the absorption map. The iteratively arrived solution to the complex object function, $O^{S}(r)$, can be represented as:

$$
O^{S}(r)=A_{O}(r) e^{i \varphi_{O}(r)}
$$

where, $A_{O}(r)$ is the amplitude function of the actinic response of the reticle and $\varphi_{O}(r)$ is the phase function (corresponding to the integral phase response of the three dimensional mask - including the absorber, multilayer stack and defects 
embedded in them). RESCAN can thus simultaneously recover both amplitude and phase responses of a three dimensionally structured EUV reticle without the need for a through-focus scan $[1,2,5]$.

Our tool and method offer several advantages over the lens-based reticle imaging methods. First, avoiding the imaging optics allows cost effective tool integration. Second, the resolution limit achievable with this method is limited merely by the NA of the pixel detector used. This is a relatively easily scalable bottleneck compared to the migration from a low NA mirror based system to its high NA counterpart. Third, the simple optical layout with a low illumination NA and a single imaging component (the pixel detector), allows through pellicle imaging. Fourth, the simultaneous recovery of the complete complex exit wave without any through focus scan, makes it possible to detect both phase and amplitude defects. In this paper, we specifically look at the sensitivity of RESCAN to phase defects by employing a sample that has programmed phase defects on a patterned EUV reticle.

\section{THE HYBRID ABSORBER-PHASE EUV RETICLE SAMPLE}

A major feature of an actinic EUV reticle inspection tool is the capability of EUV photons to penetrate within the multilayer stack and hence be sensitive to defects which photons of other wavelengths or electrons are insensitive to [12-14]. Defects embedded deep within the multilayer stack or defects on the mask blank tend to alter or leave the stack of coatings corrugated. This can lead to varied optical paths and hence induce wave front changes with little change registered in absorption image at focal plane. Capturing the effect of such defects is crucial as phase shifts due to altered multilayer morphology can have a large effect on the aerial images to the extent that absorber defects do.

To explore the phase sensitivity, we designed an EUV reticle sample with two layers. The first layer consists of a carbon pattern on a Si wafer substrate, over which multilayer coating of Si/Mo was deposited. The second layer, is an silsesquioxane (HSQ) absorber pattern on top of the multilayer coating. The carbon structure induces a phase signature in the wave that propagates and gets reflected in the corrugated multilayer stack. The details of the materials used, the vertical dimensions and the manufacturing process are described in a separate section below. The general layout of the sample that was used is provided in Fig. 4. To make a comprehensive study we designed three different samples:

1) Sample A - A low-resolution hybrid cross grating; for benchmarking purposes.

2) Sample B - An absorber pattern of logic device with programmed phase dots; To evaluate the detection performance of the tools for the phase defects for actinic patterned mask inspection.

3) Sample C - A blank sample with programmed isolated phase defects; To evaluate the performance of the tools for actinic blank inspection

To qualify the performance of RESCAN towards phase sensitive imaging, we used a hybrid cross grating structure as Sample A (see Fig.5a) for benchmarking. This consisted of a bi-layered grating structure. The phase layer is a grating with $800 \mathrm{~nm}$ half pitch and $7 \mathrm{~nm}$ tall, patterned with carbon. The absorber layer is grating with the same pitch, but perpendicular to the first layer and $140 \mathrm{~nm}$ tall HSQ was used. Orienting the gratings on both the layers in two orthogonal directions, allows independent analysis of the absorber and phase structures and the signature they leave in the actinic amplitude and phase maps.

While the cross grating is a good sample for verifying simultaneous amplitude and phase mapping, it is not a design that comes close to realistic EUV reticle fields. To verify the performance of RESCAN in a more realistic scenario, we designed another hybrid reticle, Sample B, consisting of an arbitrary absorber pattern with a CD of $200 \mathrm{~nm}$ and a matrix of phase dots of $50 \mathrm{~nm}$ diameter. The phase dots were designed to be placed under the non-absorbing zones of the top layer (see Fig.6a). The overlay error of the two layers of $10 \mathrm{~nm}$, ensured that the underlying phase dots were finally fabricated as per design and was not masked by the absorber layer.

Both the samples above were designed keeping in mind actinic imaging and metrology of patterned EUV reticles. However, towards exploring and extending the capabilities of RESCAN for metrology of actinic blanks, we designed Sample C, with a matrix of phase dots on the Si substrate, but with no absorber pattern on top layer (see Fig.7a). 

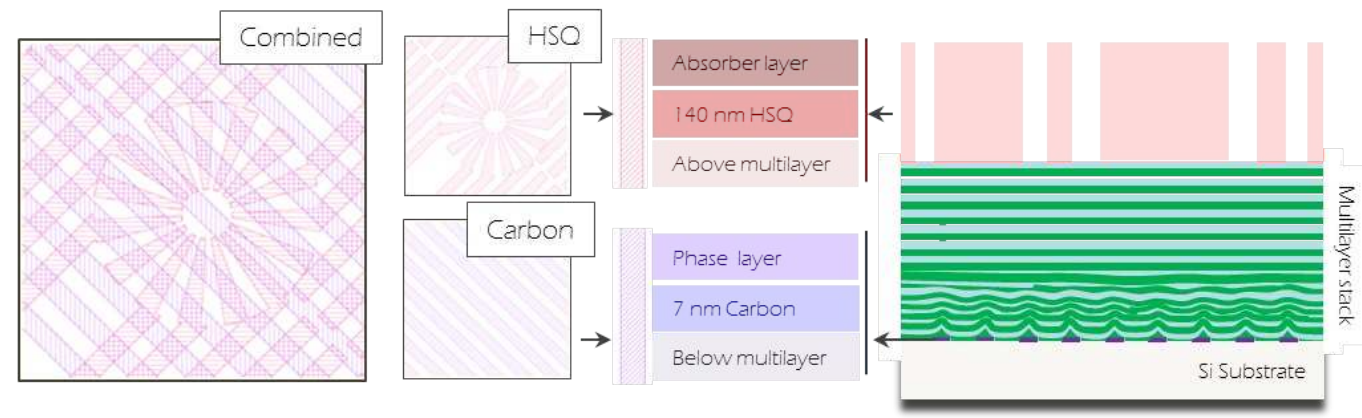

Figure 4. The hybrid sample with amplitude (red) and phase(blue) structures: (a) The top view of the sample GDS layout for the absorber layer (HSQ), phase layer(carbon) and the combined design. (b) Graphical representation of the vertical structure of the hybrid sample showing how the carbon structures on the Si substrate translates into multilayer corrugations that leads to wavefront changes and hence phase change during propagation.

The phase structures that need to be patterned beneath the multilayer were specified with (a) the intention of inducing a path difference that gives the maximum contrast and (b) to ensure that to the extent possible it's a pure phase structure with minimal absorption. Based on these design rules, we heuristically restricted the height of the phase structure to $7 \mathrm{~nm}$ ( $\sim \pi$ shift for $13.5 \mathrm{~nm}$ ), which provides multilayer ripples that on an average across the multilayer stack provides $\sim \pi$ shift in double pass for $13.5 \mathrm{~nm}[13,14]$ (This assumes a linear variation of the induced multilayer corrugations with little topology variation at the top, to $\pi$ shift at the bottom effected by the $7 \mathrm{~nm}$ high structure, leading to an average single pass phase shift of $\pi / 2$ ). The thin $7 \mathrm{~nm}$ structures at the very bottom of the multilayer also precluded absorption allowing the possibility of quasi-pure phase structures.

We fabricated the $7 \mathrm{~nm}$ structures on a Si wafer using evaporated carbon. The structures were patterned by electron beam lithography (EBL) in a positive resist layer. Subsequently the desired carbon thickness was deposited in a vacuum system by evaporating a carbon thread using short pulses. This is a controlled process that can produce thin carbon films of low roughness. The carbon structures were then defined by lift-off in acetone.

The carbon structures were imaged and characterized by atomic force microscopy (AFM), as shown in Fig. 4d. Although the height of the structures matches well with the designed height, we do observe defects at the edges of the patterns with local heights up to $30 \mathrm{~nm}$, which are due to difficulties during lift-off. The presence of these local imperfections is not expected to influence the results in this work.

The patterned Si substrate was then coated with a multilayer (ML) Mo/Si stack with a total thickness of $240 \mathrm{~nm}$ (optiX fab $\mathrm{GmbH}$, Jena, Germany). After the ML coating, the absorber patterns are defined by EBL directly into a hydrogen HSQ resist. HSQ is a negative tone, high-resolution resist that upon electron beam exposure is converted into $\mathrm{SiO}_{2}$. After the exposure and development, the HSQ structures have a thickness of $\sim 140 \mathrm{~nm}$, providing sufficient absorption at EUV wavelengths. Such HSQ absorber patterns have previously been used, showing good image contrast $[2,3,4]$.

\section{APMI OF HYBRID SAMPLES WITH RESCAN}

In this section, we show the imaging results of the samples obtained using RESCAN and we compare them with the GDS layout and the SEM images for all the three samples.

The results from the cross gratings (Sample A) are presented in Fig. 5 along with the GDS layout, the AFM image of the carbon phase structure before ML coating and the SEM images of the ML-coated, patterned sample. In the GDS layout, the stripes with the red stipple correspond to absorber patterns of HSQ and the ones with the blue stipple represent carbon structures below the multilayer. The central Siemens star in the absorber pattern is used as an alignment marker. 


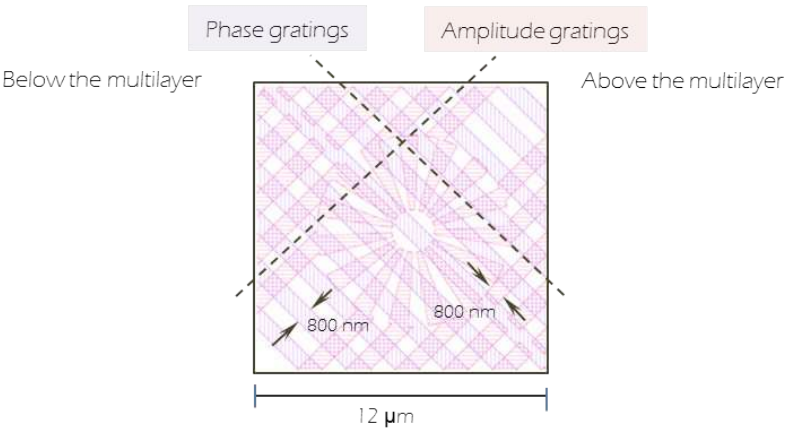

$\mathrm{b}$
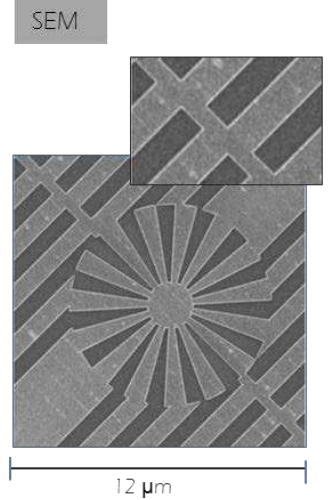

C AFM

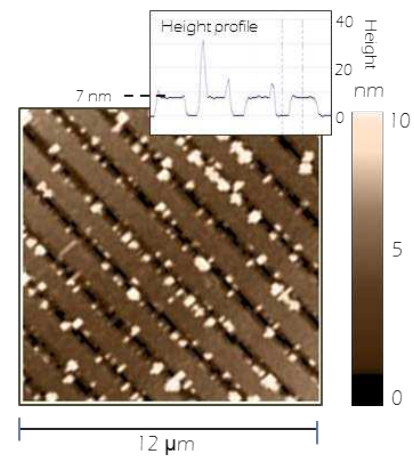

$12 \mu \mathrm{m}$

\section{RESCAN ACTINIC MAPS}

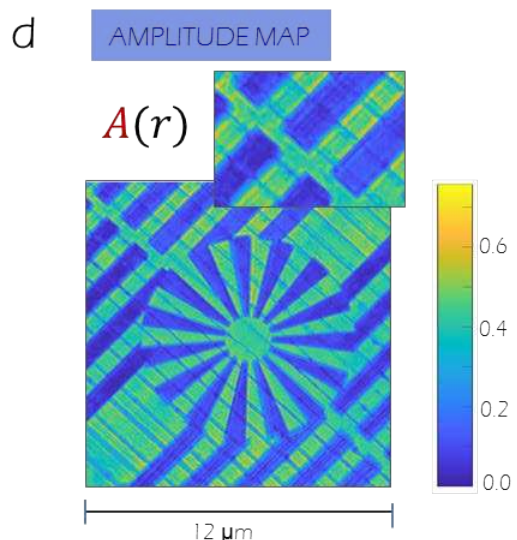

Reflects Absorption

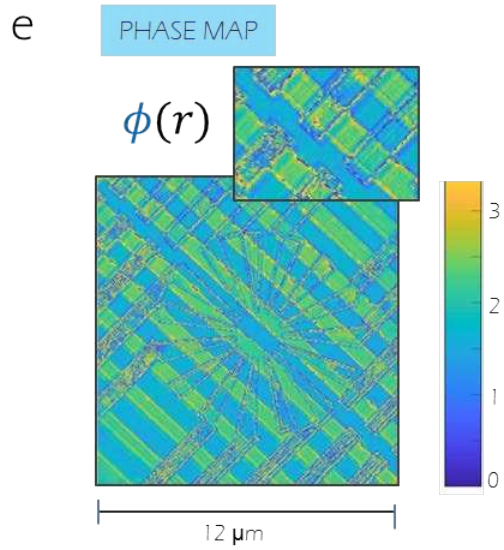

Reflects Phase -shift

Figure 5. Benchmarking results for phase imaging using the hybrid crossed-grating sample - Sample A: (a) Combined GDS layout of the absorber and phase structures ,(b) SEM image of the sample showing absorber layer contrast, (c) AFM image of the phase structures before the multilayer coating: the inset shows the one dimension height profile of a random field. One can see that the edges protrude more than the designed height of carbon owing to the lift-off error (see text), (d) actinic amplitude map of the sample, reflecting the absorber layer and the edges of the phase layer, and (e) actinic phase map, dominantly reflecting the underlying phase structures.

In the SEM image, the absorber structure is clearly seen while the signature of phase structures (the perpendicular grating) are not clearly visible. However, there is a weak edge contrast. We believe that this is due to edge defects due to imperfect 
carbon lift-off that create sharp protrusions in the multilayer. However, the main body of the grating stripes is insensitive to SEM measurements as it lies under the multilayer stack on the silicon substrate. The AFM images of the phase structure acquired before the ML coating was deposited, indicate that the carbon layer is $7 \mathrm{~nm}$ thick (see inset in Fig. 5c) in agreement with the design. The appearance of the white patches (also visible in the line profile) are once again the edge imperfections attributed to the lift-off process. Random measurements show that they could be as large as $30 \mathrm{~nm}$ pushing out onto the multilayer coating which is approximately $240 \mathrm{~nm}$ high.
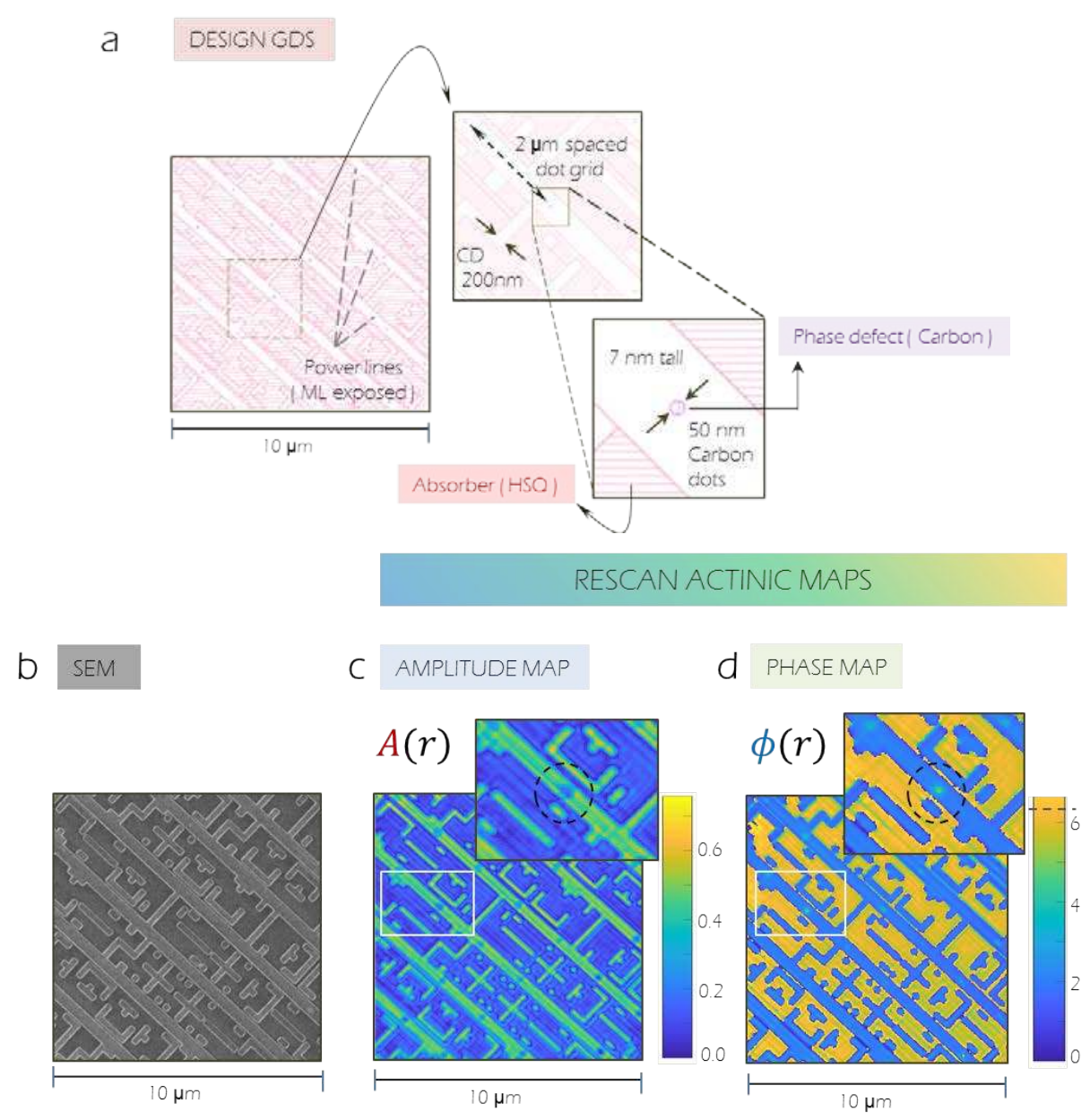

Figure 6. Results on phase dot matrix beneath a random absorber pattern - Sample B: (a) Design GDS of the combined layout of phase and amplitude. (b) The SEM image, with little sensitivity to the buried phase dots, (c) actinic amplitude image, and (d) actinic phase maps of the same field with signatures of the $50 \mathrm{~nm}$ phase dots visible within the powerlines on both RESCAN maps. The inset shows the magnified view of the zone within the white boxes. The encircled zones shows the $50 \mathrm{~nm}$ phase dot signature both in amplitude and phase maps.

The actinic images of the crossed grating hybrid sample are shown in Figs. 5d and 5e. The amplitude maps shows the absorber pattern, as seen in the SEM. The edges of the phase structure which were not clearly seen in the SEM, become prominent in the amplitude map from RESCAN, again indicative of the presence of sharper structures at the edge. The most promising result, however, is the reconstructed phase map. First, the phase grating becomes visible with a very good contrast. A second observation in the phase map is the presence of non-zero phase for the absorber layers. This is expected considering the varied optical path difference between absorber and vacuum propagation leading to phase shifts between them. The most important highlight from these preliminary results is that RESCAN can detect the phase induced by buried structures on patterned EUV masks. Our analysis shows that the phase shift induced by a carbon grating with $800 \mathrm{~nm}$ half 
pitch and $7 \mathrm{~nm}$ thickness, is around 1 radian, corresponding, for $13.5 \mathrm{~nm}$ wavelength, to about $2 \mathrm{~nm}$ effective optical path difference.

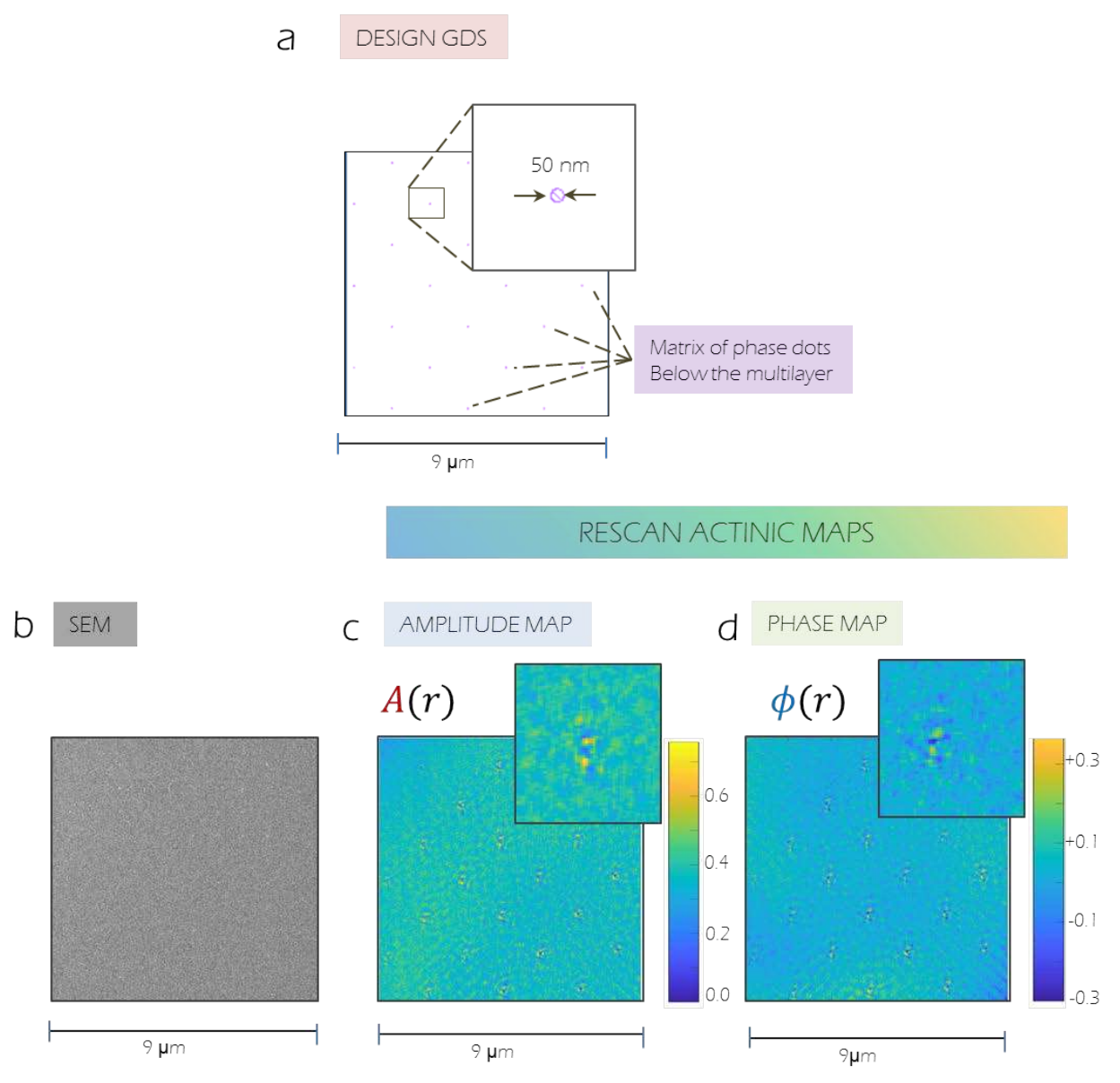

Figure 7. Results on EUV Mask Blanks - Sample C: (a) Design GDS (phase layer only), (b) SEM image, with no defects visible, (c) actinic amplitude image, and (d) phase image, showing clear signature of the programmed phase dot matrix on the Si substrate below the multilayer coating. Insets show the zoomed image of one of the phase defects in the respective images.

The imaging results from the sample with the absorber patterns similar to logic device design and with embedded phase dots are shown in Fig. 6. The GDS shows the combined layout of the absorber and the phase layers. The former in this case is an arbitrary absorber structure with a CD of $200 \mathrm{~nm}$. The phase defects, in this case, are generated by a matrix of carbon dots with a diameter of $50 \mathrm{~nm}$, a height of $7 \mathrm{~nm}$ and a pitch of $2 \mu \mathrm{m}$.

The SEM image of the field in Fig. $6 \mathrm{~b}$ shows that the phase dots are not visible as expected. The actinic amplitude and phase images are shown in Figs. 6c and 6d. Both maps show signature of the phase dots (see the insets). The amplitude map reflecting phase signature can be attributed to intensity modulation associated with wave front variation in propagation in the corrugated ML stack. Our initial analysis show that the average phase contrast of the dots from the surrounding background phase is around 0.4 radians which correspond to an optical path difference of $\sim 1 \mathrm{~nm}$. The reduced phase variation relative to the measurements on the phase gratings for the same carbon height of $7 \mathrm{~nm}$ could be attributed to the lateral sizes of the phase structure. For the cross grating sample, this structures were $800 \mathrm{~nm}$ and for the random sample with phase dots this was $50 \mathrm{~nm}$. From these measurements it is evident that the extent of observed phase shift depends not merely on the vertical height of the defects but is also sensitive to the lateral defect dimension. 
We also explored the possibility of employing RESCAN for phase defects detection on EUV mask blanks [13,14]. The programmed phase structure have $50 \mathrm{~nm}$ diameter, $7 \mathrm{~nm}$ height, and $2 \mu \mathrm{m}$ pitch. The results of the measurements on the EUV blank is shown in Fig. 7. The SEM image shows no signature of the underlying phase defects, while both the amplitude and phase actinic images clearly shows the presence of the programmed phase dots. The zoomed version of the actinic image of one of the dots shows a peculiar structure that these dots have, which was absent for the phase dots in a patterned mask. We also observe a good correlation between the structures in the amplitude and phase map. Further experiments as well as optical simulations are needed to fully understand the peculiar response of the isolated phase defects on this specific sample. The peak-to-peak phase modulation in these structures, was measured to be 0.6 radians, slightly higher than that of random sample case

\section{CONCLUSIONS AND OUTLOOK}

In this paper, we have demonstrated the phase sensitivity of the RESCAN tool for patterned masks and blank masks. By introducing programmed phase defects in EUV reticle samples, we have experimentally shown that RESCAN can detect optical path differences as small as $1 \mathrm{~nm}$ and footprint of $50 \mathrm{~nm}$. We plan to pursue the extendibility of phase defect metrology with programmed defects with smaller height and footprint. We are also envisaging experiments to quantify RESCAN's defect sensitivity in a die-to-die comparison.

\section{REFERENCES}

[1] Rajeev, R. et al., " Towards a stand-alone high-throughput EUV actinic photomask inspection tool: RESCAN," Proc. SPIE, 10145, Metrology, Inspection, and Process Control for Microlithography XXXI, 101450N. (2017).

[2] Mochi, I. et al., " Actinic inspection of EUV reticles with arbitrary pattern design," Proc. SPIE, 10450, International Conference on Extreme Ultraviolet Lithography 2017, 1045007. (2017).

[3] Mochi, I. et al., " Through-pellicle inspection of EUV masks," Proc. SPIE, 10583, Extreme Ultraviolet (EUV) Lithography IX; 105831I (2018).

[4] Bergmann, R.M. et al., " A Compact Storage Ring for the Production of EUV Radiation," Proc. International Topical Meeting on Nuclear Applications of Particle Accelerators, Quebec, (2017).

[5] Helfenstein, P. et al., " Beam drift and partial probe coherence effects in EUV reflective-mode coherent diffractive imaging," Optics Express, 26, 12242-12256, (2018).

[6] Rodenburg, J.M., et al, "A phase retrieval algorithm for shifting illumination,” Appl. Phys. Lett. 85, 4795 (2004).

[7] Thibault, P et.al," "High-resolution scanning X-ray diffraction microscopy," Science 321, 379-382 (2008).

[8] Thibault, P et.al, "Probe retrieval in ptychographic coherent diffractive imaging," Ultramicroscopy 109, 338343 (2009).

[9] Maiden, A.M, “An improved ptychographical phase retrieval algorithm for diffractive imaging," Ultramicroscopy 109, 1256-1262 (2009).

[10] Mochi, I. et al., " XIL II - RESCAN branch commissioning," , Internal Report, Advanced Lithography and Metrology Lab, PSI (2018).

[11] Elser, V. et.al, "Searching with iterated maps", PNAS 104, 418-423, (2007).

[12] Mochi, I. et.al, "Actinic imaging of native and programmed defects on a full-field mask," Proc. SPIE 7636, $76361 \mathrm{~A}(2010)$.

[13] Harada, T. et.al, "Phase imaging results of phase defect using micro-coherent extreme ultraviolet scatterometry microscope," J. Micro. Nanolithogr. MEMS MOEMS 15, 021007 (2016).

[14] Harada, T. et al, "Phase imaging of extreme-ultraviolet mask using coherent extreme-ultraviolet scatterometry microscope,” Jpn. J. Appl. Phys. 52, 06GB02 (2013). 